\title{
Robert Belot, Pierre Lamard dir., Alstom à Belfort, 130 ans d'aventure industrielle
}

Boulogne, ETAI, 2009, 377 p. + annexes.

\section{Gérard Jigaudon}

\section{(2) OpenEdition}

Journals

Édition électronique

URL : http://journals.openedition.org/dht/1870

DOI : $10.4000 /$ dht. 1870

ISSN : 1775-4194

Éditeur :

Centre d'histoire des techniques et de l'environnement du Cnam (CDHTE-Cnam), Société des élèves du CDHTE-Cnam

\section{Édition imprimée}

Date de publication : 1 décembre 2011

Pagination : 246-248

ISBN : 978-2-9530779-7-1

ISSN : 0417-8726

\section{Référence électronique}

Gérard Jigaudon, «Robert Belot, Pierre Lamard dir., Alstom à Belfort, 130 ans d'aventure industrielle », Documents pour l'histoire des techniques [En ligne], 20 | 2e semestre 2011, mis en ligne le 21 septembre 2012, consulté le 24 septembre 2020. URL : http://journals.openedition.org/dht/1870 ; DOI : https:// doi.org/10.4000/dht.1870

Ce document a été généré automatiquement le 24 septembre 2020.

(c) Tous droits réservés 


\section{Robert Belot, Pierre Lamard dir.,} Alstom à Belfort, 130 ans d'aventure industrielle

Boulogne, ETAI, 2009, 377 p. + annexes.

Gérard Jigaudon

\section{RÉFÉRENCE}

Robert Belot, Pierre Lamard dir., Alstom à Belfort, 130 ans d'aventure industrielle, Boulogne, ETAI, 2009, 377 p. + annexes. 
1 Le titre de l'ouvrage, Alstom à Belfort, suggère que l'ouvrage ne traite pas seulement de l'entreprise Alstom mais de ses rapports avec la ville qui l'héberge depuis plus d'un siècle. La réalisation $d u$ livre tient au partenariat entre la firme Alstom, la ville de Belfort, le comité interentreprises Trois-Chênes et l'Université de Technologie de BelfortMontbéliard (UTBM). Loin de la monographie hagiographique d'entreprise ou d'usine, ce recueil est dirigé par deux historiens, enseignants à

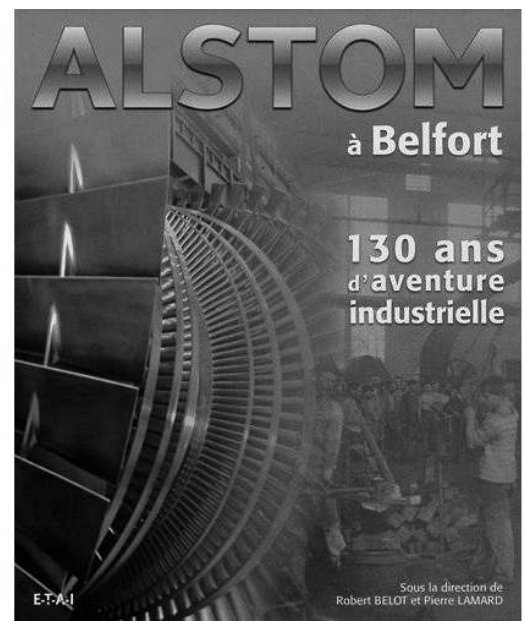
l'UTBM, bien connus pour leur activité au sein du laboratoire RECITS, à qui l'on doit déjà Peugeot à Sochaux : des hommes, une usine, un territoire et qui se sont entourés de chercheurs en histoire, spécialistes de la région.

2 C'est en 1879 que l'usine de Belfort voit le jour dans les conditions dont on connaît les grandes lignes. A la suite de la défaite de 1870, la France perd les ressources industrielles implantées en Alsace et Lorraine. C'est le cas notamment de la Société alsacienne de constructions mécaniques (SACM) créée en 1872 après le regroupement des usines de Mulhouse de la société André Köchlin avec les usines de Graffenstaden. Elle est alors la plus importante firme de constructions mécaniques (locomotives surtout, mais aussi machines textiles, moteurs, transmissions, etc.) d'Alsace. Mais elle se trouve désormais en Allemagne intégrée dans le Zollverein alors que ses clients principaux sont dans une France qui renforce son protectionnisme en pratiquant des droits de douane élevés. C'est à ce moment que s'annonce un formidable marché avec l'adoption en France du plan Freycinet lancé en 1878, qui prévoit la construction de $17000 \mathrm{~km}$ de voies ferrées en complément du réseau existant. C'est sans doute dans cette conjoncture que naît l'idée de l'implantation belfortaine de ce qui au départ ne doit être qu'un atelier d'assemblage des pièces usinées dans les usines alsaciennes.

Le choix de Belfort est évident : la ville n'est qu'à quelques dizaines de kilomètres de Mulhouse. Elle fait partie du département du Haut-Rhin avant la guerre de 1870 et grâce à la résistance conduite par Denfert-Rochereau, elle reste française. De nombreux alsaciens ayant refusé l'occupation allemande - les optants - s'y sont réfugiés, la population a presque triplé entre 1867 et 1872, la main d'œuvre y est donc abondante. Les liaisons par chemin de fer, route, canal ne manquent pas avec Mulhouse, comme avec le reste de la France. La greffe prend rapidement, dès 1880 les ateliers s'élèvent les uns après les autres, les fabrications se diversifient, les embauches se multiplient, des structures de formation sont mises en place et l'habitat ouvrier s'étend. C'est ce prodigieux essor qui nous est conté, mais aussi les paliers dans la croissance, comme les périodes des deux guerres mondiales ou les crises récentes dont l'une a même eu raison de la commémoration du centenaire de l'usine.

$4 \quad$ L'abondance et la qualité des sources ont conduit les auteurs à nous offrir un ouvrage remarquable par son volume mais aussi par l'exceptionnelle richesse de ses illustrations : plans du site, dessins industriels et photographies. L'ouvrage retrace plus d'un siècle d'innovations technologiques mais aussi l'empreinte d'une entreprise sur un 
territoire et ses habitants. Ces derniers sont d'ailleurs largement mis à contribution et leurs témoignages ne font pas partie des aspects les moins passionnants du livre.

5 La partie purement ferroviaire de l'ouvrage et la construction de locomotives en particulier est assez conséquente et occupe un peu plus de soixante pages, soit une proportion sensiblement équivalente à celle de l'activité transport sur le site de Belfort en 2005. On sera surpris d'apprendre que la SACM utilisait pour désigner ses locomotives à vapeur le système des chiffres pour les essieux porteurs et celui des lettres pour les essieux moteurs. C'est-à-dire celui employé par la SNCF pour les locomotives diesel et électriques. Une locomotive à deux essieux porteurs à l'avant, un à l'arrière et trois essieux moteurs au centre était donc désignée $2-\mathrm{C}-1$ et non 231 selon la terminologie courante (pour les anglo-saxons ce serait une 4-6-2). Ces engins méritaient assez peu le qualificatif de "mastodontes" car il s'agissait de locomotives relativement légères et élégantes destinées aux trains les plus rapides. Le terme s'appliquait mieux aux 150 ou decapod pour trains de marchandises mais là encore on est loin des big boys américaines.

6 Les auteurs n'hésitent pas, bien que de manière discrète, à écorner la vérité officielle concernant le record de vitesse de mars 1955 en reproduisant la une de trois quotidiens régionaux du 29 mars et en mentionnant entre parenthèses : « en fait, il semblerait que la BB (9004, construite au Creusot) soit un peu plus rapide (que la CC 7107 d'Alsthom), mais personne alors n'en souffle mot: il ne faut pas froisser les constructeurs! ». Cette attitude perdurera longtemps puisqu'en 2005 lors du cinquantenaire du record des Landes, ni la SNCF ni Alstom ne sont revenus sur la version idéaliste du record partagé à égalité.

7 Le passage relatif à la participation d'Alsthom aux débuts du métro sur pneu est un peu confus (p. 109). L'entreprise a bien participé à la mise au point de la motrice MP 51 familièrement connue sous le nom de grand-mère - dont elle a fourni les moteurs, mais celle-ci n'a jamais circulé que sur la navette Porte des Lilas - Porte du Pré-Saint-Gervais de 1952 à 1956. C'est à la suite des résultats probants de ces essais que la RATP a décidé d'équiper la ligne $\mathrm{n}^{\circ} 11$, Mairie des Lilas - Châtelet de rames sur pneumatiques dont Alsthom a fourni une partie des moteurs et des bogies.

Une légende de photo (p. 119) présente la locomotive X 996 d'Amtrak (exploitant ferroviaire des services voyageurs aux États-Unis). Il ne s'agit pas d'une locomotive destinée à l'exportation mais d'une des quatre CC 21000 de la SNCF - la 21003 - envoyée comme engin de démonstration entre New-York et Washington. Elle y a effectué une campagne d'essais peu fructueuse puisque c'est le constructeur suédois ASEA qui a finalement emporté le marché américain. Elle a été remise au type à son retour en France.

Certes le TGV a été à la source d'un grand nombre d'innovations en matière de traction électrique mais il faut préciser que le fil de contact de la caténaire a toujours été suspendu en zigzag au dessus de la voie afin d'éviter une usure de la lame du pantographe au même endroit dans les lignes droites. Quant au pantographe appelé unijambiste il est apparu dès le milieu des années 1960. La caractéristique du pantographe du TGV Paris Sud-Est était d'être à double étage. C'est la solution qui avait été trouvée à l'époque pour résoudre le problème de la captation du courant à grande vitesse car le contact doit être constant - mais jamais appuyé - entre la lame du pantographe et le fil (p. 129). 
10 On peut regretter que l'accent ne soit pas mis sur les dernières productions de la marque, c'est-à-dire la locomotive électrique Prima II (p. 124) et l'AGV (Automotrice à Grande Vitesse - et non "autorail ») (p. 138). Ces deux engins ayant pour caractéristiques communes d'avoir été développés par Alstom sur ses fonds propres et d'avoir déjà fait l'objet de commandes étrangères : l'office national des chemins de fer marocains (ONCM) dans le premier cas, l'opérateur privé italien Nuovo Trasporto Viaggiatori (NTV) dans le second. On peut ajouter que l'AGV est le premier train à grande vitesse au monde à concilier l'articulation du TGV français et la motorisation répartie de l'ICE allemand.

11 Le chapitre ferroviaire " La conquête de la vitesse de la vapeur au TGV » se termine par un paragraphe intitulé «Le paradoxe belfortain » (p. 138). En effet, Belfort qui a vu sortir tous les types de TGV en service en France n'est pas située sur une ligne à grande vitesse. Cet "oubli » sera bientôt réparé puisque le chantier de la LGV Rhin-Rhône avance à grands pas. En 2011, Belfort aura sa gare TGV reliée - une fois n'est pas coutume - au réseau classique après réouverture de la ligne Belfort - Delle. Elle verra passer - et s'arrêter - des trains en provenance de Strasbourg et d'Allemagne à destination du Midi et de l'Espagne, ainsi que d'autres reliant Paris à Bâle et à Zurich.

\section{AUTEURS}

GÉRARD JIGAUDON

CDHTE - Cnam 